\title{
ISOTHERMAL ENTHALPY AND DENSITY STUDIES \\ OF POLYMERIC GLASSES VERSUS FORMATION PARAMETERS
}

\author{
Frank E. Filisko \\ Department of Materials and Metallurgical Engineering \\ University of Michigan \\ Ann Arbor, Michigan 48109
}

Time-dependent properties of glassy polymers have been studied extensively by observing changes with time of either specific volume or "apparent enthalpy" obtained from scanning-calorimeter-derived specific heats. It is known, however, that the rate of changes of these parameters are dependent not only on temperature but as well on the initial state of the glass;' yet few studies have been directed toward systematically correlating more than one processing parameter (commonly, annealing temperature) with more than one ordering parameter. Likewise, little attention has been directed toward the initial state of the glass. In this study we independently control the vitrification pressure $\left(P_{v}, 15\right.$ to $\left.60,000 \mathrm{psi}\right)$ and vitrification cooling rate $\left(R_{v}, 0.1^{\circ}\right.$ to $\left.400^{\circ} \mathrm{K} / \mathrm{min}\right)$ and determine enthalpies measured isothermally $\left(\Delta h_{r}\right),{ }^{2}$ densities, and qualitatively compare scanning calorimeter traces. We observe that, for polystyrene at time $=0, \Delta h_{r}$ increases linearly and strongly with $\log R_{v}$ and increases linearly but weakly with $P_{v}$. Likewise, we observe that density decreases linearly with $\log R_{v}$. Most important is the fact that the pressure and cooling rate coefficients of both $\Delta h_{r}$ and density are virtually independent, i.e., $\partial \Delta h_{r} / \partial P_{\nu}$ and $\partial \rho / \partial P_{\nu}$ are independent of $R_{\nu}$, and $\partial \Delta h_{r} / \partial R_{v}$ and $\partial \rho / \partial R_{v}$ are independent of $P_{v}$. Thus we can write:

$$
\Delta h_{r}=\Delta h_{r 0}+\mathrm{A} \log R_{v}+B P_{v}
$$

and

$$
\rho=\rho_{0}+C \log R_{v}+D f\left(P_{v}\right),
$$

where $A, B, C, D$ are constants and $f\left(P_{v}\right)$ is a function of the form $\left[1+\exp \left(-1 / P_{v}\right)\right]$. If we eliminate $\log R_{v}$ from both equations we obtain an expression equating the parameters $\Delta h_{r}, \rho$, and $P_{v}$; only two being independent. The uniqueness of this is related to the isothermally obtained enthalpies which are unambiguous in their interpretation. They are of particular value in studying the glassy state because:

1. Enthalpy is a well-defined fundamental thermodynamic parameter.

2. The enthalpies are determined at a constant temperature, important in studying glasses because of the temperature-dependent relaxations.

3. Time can be known exactly up to the start of the measurement.

The method involves techniques of solution calorimetry. ${ }^{2}$ This presentation involves only the results at time-0, but relaxation studies are going on. Further, scanning calorimeter traces were obtained on all samples, the results clearly demonstrating that the endotherm superimposed on $T_{g}$ (which has become associated with enthalpy relaxation) is a strong function of density as well as presumably enthalpy. 


\section{REFERENCES}

1. Kovacs, A. J., J. J. Aklonis, J. M. Hutchinson \& A. R. Ramos. 1979. J. Polym. Sci. Phys. 17: 1097 .

2. Filisko, F, E., H. T. Kau, J. DeRudder \& J. Golba. 1977. In Structure-Solubility Relationships in Polymers. F. W. Harris \& R. B. Seymour, Eds. p. 59. Academic Press, New York, N.Y. 